\title{
Effects of sevoflurane general anesthesia during early pregnancy on AIM2 expression in the hippocampus and parietal cortex of Sprague-Dawley offspring rats
}

\author{
YULIN ZHU ${ }^{1 *}$, CHAO LV ${ }^{2 *}$, JINGYING LIU $^{3}$, SHUJUN SHANG ${ }^{1}$ and WEI JING ${ }^{4}$ \\ ${ }^{1}$ Department of Anesthesiology, Yantaishan Hospital, Yantai, Shandong 264000; ${ }^{2}$ Department of Anesthesiology, \\ People's Hospital of Laixi, Laixi, Shandong 266600; ${ }^{3}$ Department of Obstetrics, Yantaishan Hospital, Yantai, \\ Shandong 264000; ${ }^{4}$ Department of Anesthesiology, Taian City Central Hospital, Taishan, Taian, Shandong 271000, P.R. China
}

Received April 24, 2020; Accepted December 10, 2020

DOI: $10.3892 /$ etm.2021.9900

\begin{abstract}
The aim of the present study was to investigate the effect of exposure to sevoflurane general anesthesia during early pregnancy on interferon-inducible protein AIM2 (AIM2) expression in the hippocampus and parietal cortex of the offspring Sprague-Dawley (SD) rats. A total of 18 SD rats at a gestational age of 5-7 days were randomly divided into three groups: i) A control group (control); ii) 2-h sevoflurane general anesthesia, group 1 (S1); and iii) 4-h sevoflurane general anesthesia, group 2 (S2). The six offspring rats in each group were maintained for 30 days and assessed by Morris water maze testing. Brain specimens were collected from offspring rats 30 days after birth. Changes in the structural morphology of neurons in the hippocampus and parietal cortex were observed using hematoxylin and eosin staining. Nissl bodies in the hippocampus and parietal cortex were observed by Nissl staining. The expression of glial fibrillary acidic protein (GFAP), AIM2, CD45 and IL-1 $\beta$ was detected by immunohistochemistry and the protein levels of CD45, IL-1 $\beta$, pro-caspase- 1 and caspase- 1 p10 were detected by western blotting. Compared with the control group, offspring rats in the S1 and S2 groups exhibited poor long-term learning and memory ability and experienced different degrees of damage to both the hippocampus and parietal cortex. The expression levels of GFAP, AIM2, CD45, IL-1 $\beta$, caspase-1 and caspase-1 p10 in the offspring of both the $\mathrm{S} 1$ and the $\mathrm{S} 2$ groups were significantly increased $(\mathrm{P}<0.05)$ compared with offspring of the control group. Moreover, compared with the offspring
\end{abstract}

Correspondence to: Dr Wei Jing, Department of Anesthesiology, Taian City Central Hospital, 29 Longtan Road, Taishan, Taian, Shandong 271000, P.R. China

E-mail: wmfsg65@163.com

${ }^{*}$ Contributed equally

Key words: early pregnancy, sevoflurane general anesthesia, hippocampus, parietal cortex, interferon-inducible protein AIM2 of the S1 group, hippocampal and parietal cortex injury in the offspring of the S2 group was further aggravated, and the expression of GFAP, AIM2, CD45, IL-1 $\beta$, pro-caspase-1 and cleaved-caspase-1 was significantly increased $(\mathrm{P}<0.05)$. In conclusion, sevoflurane general anesthesia in SD rat early pregnancy promoted the expression of AIM2 and the inflammatory response in the hippocampus and parietal cortex of offspring rats.

\section{Introduction}

In recent years, sevoflurane has become one of the most commonly used anesthetics for medical procedures during pregnancy (1). A previous investigation has determined the efficacy and safety of sevoflurane in different organ systems and patient populations (2). A wide range of studies have suggested that during brain development, a long-term risk of neurobehavioral disorders and neurodegeneration is apparent following exposure to anesthetics $(3,4)$, and that the concentration of sevoflurane plays an important role in the process of neurodevelopmental damage (5). Consequently, the administration of anesthetic drugs during early brain development may result in neural dysfunction and damage (6).

The common consensus is that exposure to anesthetics during pregnancy is likely to affect fetal brain development, thus the methods used to assess the safety of surgery and anesthesia are primarily based on animal models (7). As an inhaled anesthetic with a rapid recovery profile, sevoflurane is prevalently used worldwide. Human neural development is similar to that of rats, and studies of model animals are helpful in research and the discovery of novel therapeutic options $(8,9)$. Although previous studies have described the efficacy and safety of sevoflurane, the present study primarily focused on the effects of exposure duration on neurodevelopment $(10,11)$. Due to the differences in drug responses, doses and time-scales of embryological development, as well as a number of other confounding variables, the conclusions drawn by retrospective human studies can be difficult to interpret.

A previous study has reported that sevoflurane-induced neurotoxicity promotes changes in the development of the hippocampus (12). The first trimester is a stage at which there 
are high levels of neurogenesis throughout the cortex, and the development of the hippocampus, which is responsible for high-level cognitive functions, plays important roles in the onset and development of various neurodevelopmental defects (13).

In the present study, the duration of sevoflurane general anesthesia in the hippocampus and parietal cortex was assessed in early pregnancy. The expression of marker genes such as interferon-inducible protein AIM2 (AIM2) and CD45 was detected to reveal neuronal cell changes. The protein levels of glial fibrillary acidic protein (GFAP), AIM2, CD45, IL-1 $\beta$, pro-caspase- 1 and cleaved-caspase- 1 were used to further verify these results.

\section{Materials and methods}

Animals. A total of 18 Sprague-Dawley (SD) rats were purchased from Beijing Weitong Lihua Experimental Animal Technology Company Co., Ltd. [license number SCXK (Beijing) 20160006]. The rats were of specific-pathogen free grade and at the gestational age of 5-7 days (weight, $250 \pm 30 \mathrm{~g}$ ). The animals were housed at $23 \pm 2^{\circ} \mathrm{C}, 55 \pm 5 \%$ humidity with a 12-h light/dark cycle, and with free access to food and water. All animal experiments were conducted following the National Institutes of Health (NIH) guidelines (14) and were reviewed and approved by the Yantaishan Hospital Animal Protection and Use Committee (approval no. 2018-10087). Animal health and behavior were monitored every day, including assessment of diet, weight, mental states and mortality.

Animal grouping. Pregnant SD rats were randomly divided into 3 groups ( $n=6 /$ group): The control group (control), sevoflurane general anesthesia group 1 (S1) and sevoflurane general anesthesia group 2 (S2).

Sevoflurane exposure. Rats in the control group were untreated, and those in the two sevoflurane exposure groups (S1 and S2) were exposed to $2 \%$ sevoflurane $(15-18)$. All rats were placed in separate cages and treated using $100 \%$ oxygen as a carrier gas, with a 4 1/min total gas flow. Rats in the S1 and S2 group were anesthetized with $5 \%$ sevoflurane for 1-2 min until they became unconscious. The $2 \%$ sevoflurane was used for maintenance, and anesthesia was performed for 2 and $4 \mathrm{~h}$ in S1 and S2 groups, respectively. During sevoflurane anesthesia, the concentrations of oxygen, carbon dioxide and sevoflurane in the anesthesia chamber were monitored using a gas detector (Drägerwerk AG \& Co. KGaA). Sevoflurane exposure was stopped at the indicated time points and the pregnant rats were returned to their cages until giving birth. In the control group, there were 35 female offspring rats and 28 male offspring rats. In the $\mathrm{S} 1$ group, there were 32 female offspring rats and 33 male offspring rats. In the S2 group, there were 29 female offspring rats and 34 male offspring rats. The animals were housed at $23 \pm 2^{\circ} \mathrm{C}, 55 \pm 5 \%$ humidity with a 12 -h light/dark cycle, and with free access to food and water. A total of 30 days post-birth, 12 offspring (male:female ratio 1:1) rats (weight, 110 $\pm 20 \mathrm{~g}$ ) were randomly selected from each group, anesthetized by an intraperitoneal injection of $3 \%$ sodium pentobarbital $(50 \mathrm{mg} / \mathrm{kg})$ and sacrificed by decapitation. Five minutes after cardiac arrest, death was confirmed. Brain specimens were then collected. The brain samples of six rats/group were fixed in $4 \%$ paraformaldehyde for $24 \mathrm{~h}$ at $4^{\circ} \mathrm{C}$, and those from the other six rats/group were snap frozen and stored in liquid nitrogen for western blot analysis. In each group, 18 offspring rats were euthanized. No animals died during the experiment.

Morris water maze test. A total of 6 offspring rats were randomly selected from each group and maintained for 30 days prior to Morris water maze testing. A round swimming pool (diameter, $180 \mathrm{~cm}$; depth, $60 \mathrm{~cm}$ ) was prepared with a water temperature of $23 \pm 1^{\circ} \mathrm{C}$. An underwater platform (diameter, $10 \mathrm{~cm} ; 2 \mathrm{~cm}$ below the water surface) was placed in the first quadrant of the pool. Testing was initiated at 9 a.m. on the 30th day after birth, and lasted for 5 days, with 4 tests/day. The rats were placed into the water at the designated release point and allowed $90 \mathrm{sec}$ to locate the platform. After remaining on the platform for $15 \mathrm{sec}$, the rats were removed from the pool and the experiment was terminated. If the rats failed to locate the platform within $90 \mathrm{sec}$, they were manually placed on the platform for $15 \mathrm{sec}$. Swimming time and speed were recorded and the platform was removed to conduct the probe experiment, as previously described (19).

Hematoxylin and eosin $(H \& E)$ and Nissl staining. After dehydration, the brain specimens were fixed in $4 \%$ paraformaldehyde solution, as aforementioned, and embedded in paraffin. Paraffin blocks of brain tissue included sections of the hippocampus and parietal cortex (5- $\mu \mathrm{m}$ thick). The sections were routinely dewaxed with xylene at $60^{\circ} \mathrm{C}$ and hydrated using a graded ethanol series. The tissues were stained with hematoxylin (Sigma-Aldrich; Merck KGaA) for $5 \mathrm{~min}$ at room temperature, and then rinsed with tap water. The tissues were then differentiated for $30 \mathrm{sec}$ using hydrochloric acid and ethanol, immersed in tap water for $15 \mathrm{~min}$ at room temperature, and then placed in eosin staining solution (Sigma-Aldrich; Merck KGaA) for $2 \mathrm{~min}$ at room temperature. All samples were routinely dehydrated and sealed. For Nissel staining, the sections were incubated at $37^{\circ} \mathrm{C}$ overnight, rehydrated and then subjected to Nissl staining for $10 \mathrm{~min}$ at room temperature. These samples were also dehydrated, cleared and sealed. The morphology of neurons in the hippocampus and parietal cortex was observed using a light microscope at x400 magnification (Olympus BX51; Olympus Corporation).

Immunohistochemistry. After conventional sectioning of the hippocampal and parietal cortex tissues ( $5 \mu \mathrm{m}$ thick), the specimens were dewaxed with xylene at $60^{\circ} \mathrm{C}$ and hydrated with a graded series of ethanol solutions. The sections were inactivated using $3 \% \mathrm{H}_{2} \mathrm{O}_{2}$ for $20 \mathrm{~min}$ at room temperature, fixed in citrate buffer ( $\mathrm{pH}$ 6.0) with high-temperature heating to boiling for $10 \mathrm{~min}$, and then treated with 5\% BSA (cat. no. SW3015: Beijing Solarbio Science \& Technology Co., Ltd.) for $20 \mathrm{~min}$ at room temperature. Rabbit anti-rat GFAP (1:800; cat. no. orb10706), AIM2 (1:200; cat. no. orb45726), CD45 (1:300; cat. no. orb10328) and IL-1 $\beta$ (1:300; cat. no. orb499934) (all Biorbyt Ltd.) were added and the tissues were incubated at $4^{\circ} \mathrm{C}$ overnight. After rewarming, the specimens were incubated with goat anti-rabbit horseradish peroxidase $\operatorname{IgG}(1: 1,000$; cat. no. ABIN101988; Antibodies Online GmbH). The sections were developed with 3,3'-diaminobenzidine, followed by counterstaining with hematoxylin for $10 \mathrm{~min}$ at room temperature, dehydration, clearing and sealing. The relevant brain regions 
(5 randomly selected fields per sample) were observed under a light optical microscope (Olympus Corporation) at x400 magnification, and cells were counted using Aperio ImageScope 11.1 software (Leica Microsystems, Inc.).

Western blotting. Sections of the hippocampus and parietal cortex were ground and homogenized using a total protein extraction kit (cat. no. BC3710; Beijing Solarbio Science \& Technology Co., Ltd.) according to the manufacturer's instructions. After centrifugation at $12,000 \mathrm{x} \mathrm{g}$ for $10 \mathrm{~min}$ at $4^{\circ} \mathrm{C}$, the supernatant was removed and a BCA kit was used to determine protein concentration (Beijing Solarbio Science \& Technology Co., Ltd.). A total of $40 \mu \mathrm{g}$ protein/lane was separated by SDS-PAGE $(10 \%$ gel) in a 1:1 dilution with $5 \mathrm{X}$ protein loading buffer (cat. no. P1040; Beijing Solarbio Science \& Technology Co., Ltd.) and heated at $95^{\circ} \mathrm{C}$ for $5 \mathrm{~min}$ to denature the protein. The samples were then transferred to PVDF membranes (Merck KGaA) at a voltage of $80 \mathrm{~V}$ for $30 \mathrm{~min}$ and blocked with a TBS with $0.1 \%$ Tween-20 (TBST) solution containing 5\% skimmed milk powder for $1 \mathrm{~h}$ at $4^{\circ} \mathrm{C}$. Rabbit anti-rat CD45 (1:1,000; cat. no. orb10328; Biorbyt Ltd.), IL-1ß (1:1,000; cat. no. orb499934; Biorbyt Ltd.), caspase-1 (1:800; cat. no. PA5-86936; Thermo Fisher Scientific, Inc.), caspase-1 p10 (1:1,000; cat. no. PA5-39882; Thermo Fisher Scientific, Inc.) and $\beta$-actin (1:2,000; cat. no. orb178392; Biorbyt Ltd.) polyclonal antibodies were diluted with a TBST solution containing $3 \%$ bovine serum protein (cat. no. ab64009; Abcam) and used to probe the membranes at $4^{\circ} \mathrm{C}$ overnight. After rewarming, the membranes were incubated with goat anti-rabbit horseradish peroxidase-labeled $\operatorname{IgG}(1: 1,000$; cat. no. ABIN101988; Antibodies Online $\mathrm{GmbH}$ ) for $1 \mathrm{~h}$ at room temperature and washed with ECL (cat. no. PE0010; Beijing Solarbio Science \& Technology Co., Ltd.) for 3-5 min. The protein levels were normalized to those of $\beta$-actin and grayscale scanning and semi-quantification were performed using ImageJ 1.8 software (NIH).

Statistical analysis. The data were processed using SPSS 19.0 software (IBM Corp.) and are presented as the mean \pm SEM. The number of repeats was 6. Statistical significance was determined by ANOVA followed by Tukey's test for multiple comparisons. The values of swimming speed and escape latency were analyzed using one-way repeated-measures ANOVA followed by Bonferroni post hoc analysis. $\mathrm{P}<0.05$ was considered to indicate a statistically significant difference.

\section{Results}

Effects of sevoflurane general anesthesia in early pregnancy on the learning ability of offspring rats. In order to study the effect of sevoflurane general anesthesia in early pregnancy on the long-term learning and memory of offspring rats, Morris water maze tests were performed on each group of rats. The results showed no significant differences in the activity speed of each group of rats (Fig. 1A). Compared with the control group, the escape latency of the rats in the S1 and S2 group increased on days 3-5 of training $(\mathrm{P}<0.05)$, and the number of crossings of the original platform decreased significantly (Fig. 1B and C; $\mathrm{P}<0.05$ ). Compared with the $\mathrm{S} 1$ group, the $\mathrm{S} 2$ group exhibited poorer learning ability, the escape latency of the rats in the S2 group increased on day 2 of training (Fig. 1B; $\mathrm{P}<0.05$ ).
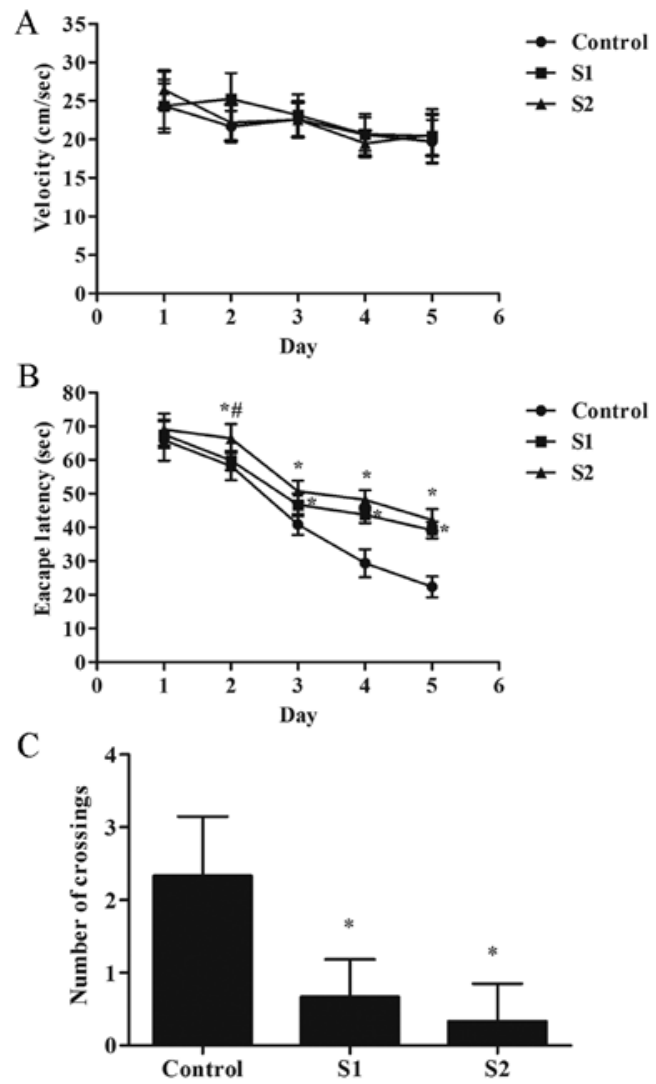

Figure 1. Behavior of offspring rats as determined by Morris water maze testing. (A) Swimming speed. (B) Escape latency. (C) Exploratory experiment. ${ }^{\prime} \mathrm{P}<0.05$ vs. the control group; ${ }^{\prime} \mathrm{P}<0.05$ vs. the $\mathrm{S} 1$ group. $\mathrm{S} 1,2-\mathrm{h}$ sevoflurane general anesthesia; S2, 4-h sevoflurane general anesthesia.

Effects of sevoflurane general anesthesia during early pregnancy on the morphology of hippocampal and parietal cortex neurons in offspring rats. The objective of the present study was to characterize the effects of sevoflurane general anesthesia in early pregnancy on offspring rats. The morphological changes of neurons in the hippocampus and parietal cortex were observed by $\mathrm{H} \& \mathrm{E}$ and Nissl staining. The H\&E staining results showed that the hippocampus and parietal cortex neurons of offspring rats in the control group were uniform in size, arranged neatly and exhibited a clear outline (Fig. 2A). When compared with the control group, the neuronal cells of the S1 group were arranged relatively neatly, and a small number showed swelling and neuronal degeneration. However, cells from the $\mathrm{S} 2$ group were arranged in a disordered manner, the cell bodies were shrunken and the nuclei were condensed into triangles or polygons. The Nissl staining results illustrated that the hippocampus and parietal cortex of offspring rats in the control group had normal, clear and complete Nissl bodies (Fig. 2B). When compared with the control group, the Nissl bodies appeared diffuse in the $\mathrm{S} 1$ and $\mathrm{S} 2$ group. In the $\mathrm{S} 2$ group, the Nissl bodies appeared more diffuse than in the S1 group.

Effects of sevoflurane general anesthesia during early pregnancy on the expression of GFAP and AIM2 in hippocampal and parietal cortex neurons in offspring rats. The expression of GFAP in offspring rats was evaluated to determine the effects of sevoflurane on the hippocampus and parietal cortex. Compared with the control group, the expression of 


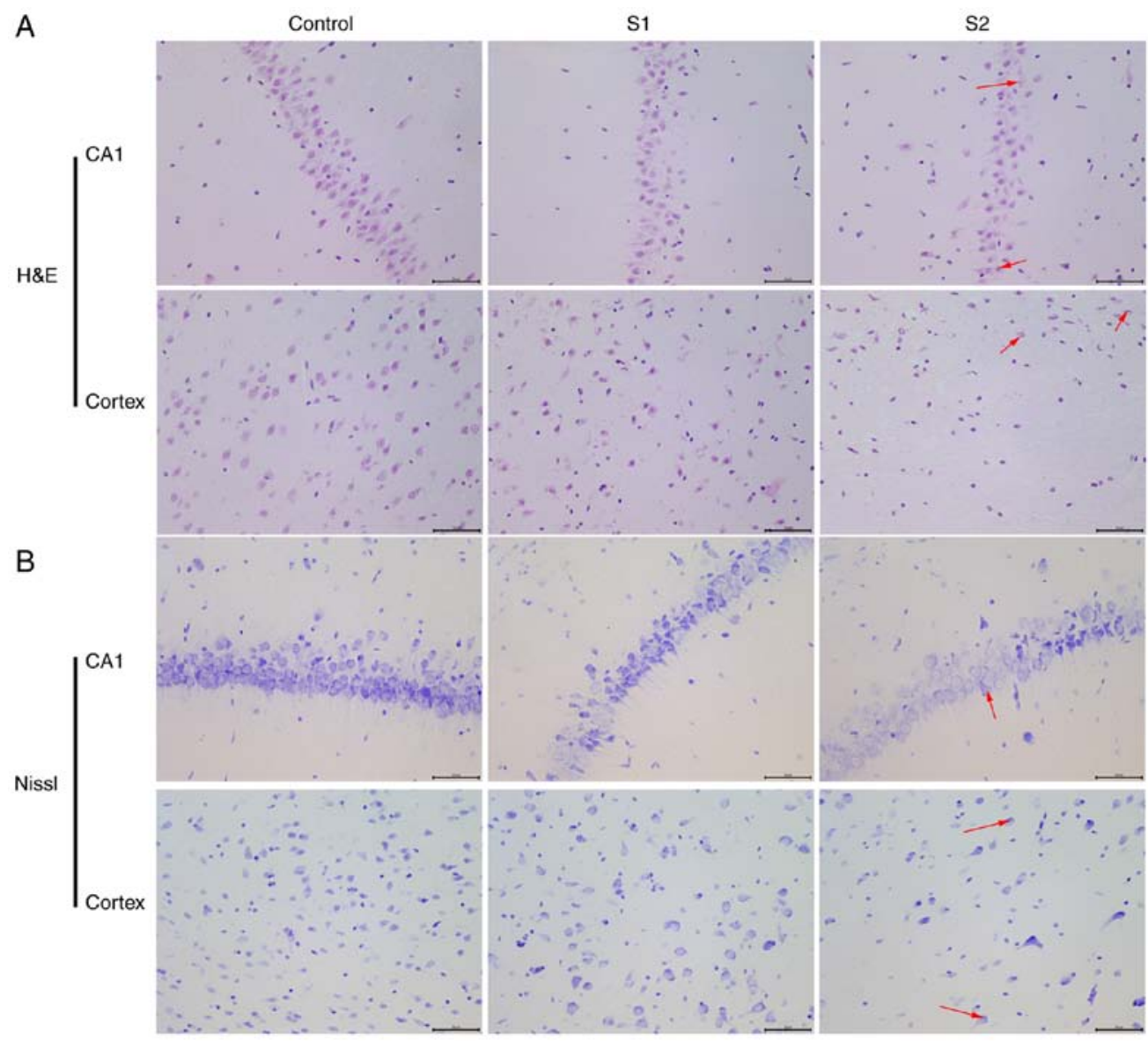

Figure 2. Morphological changes to CA1 and cortex neurons. (A) H\&E and (B) Nissl staining. Scale bar, $50 \mu \mathrm{m}$. CA1, hippocampal cornu ammonis 1; cortex, parietal cortex; S1, 2-h sevoflurane general anesthesia; S2, 4-h sevoflurane general anesthesia; H\&E, hematoxylin and eosin.
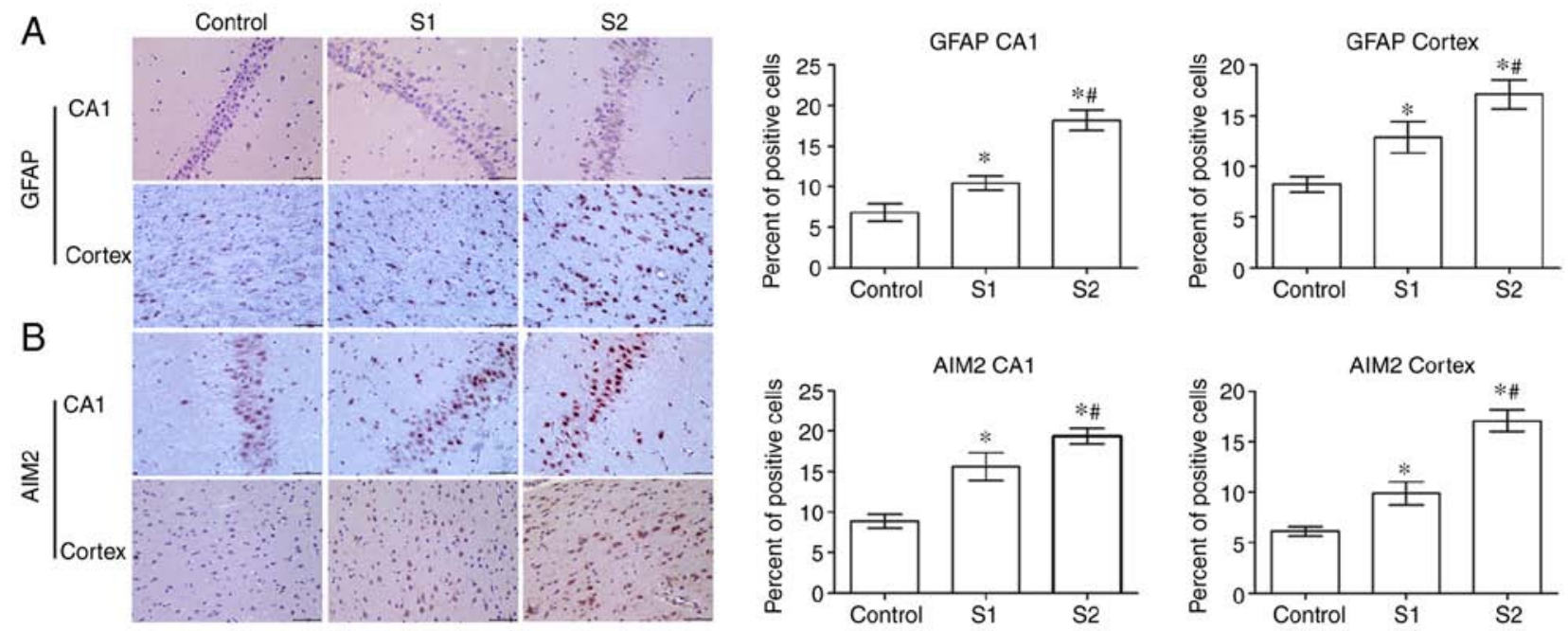

Figure 3. Immunohistochemical analysis of (A) GFAP and (B) AIM2 expression in the CA1 and cortex. Scale bar, $50 \mu \mathrm{m}$. ${ }^{*} \mathrm{P}<0.05$ vs. the control group; ${ }^{*} \mathrm{P}<0.05$ vs. the S1 group. GFAP, glial fibrillary acidic protein; AIM2, interferon-inducible protein AIM2; CA1, hippocampal cornu ammonis 1; cortex, parietal cortex; S1, 2-h sevoflurane general anesthesia; S2, 4-h sevoflurane general anesthesia.

GFAP in the S1 and S2 groups was significantly increased $(\mathrm{P}<0.05)$ in the hippocampus and parietal cortex, and GFAP expression was higher in the $\mathrm{S} 2$ group than in the $\mathrm{S} 1$ group $(\mathrm{P}<0.05$; Fig. 3A). Further experiments demonstrated that the expression of AIM2 in the S1 and S2 groups was significantly increased compared with the control group $(\mathrm{P}<0.05)$.
Furthermore, the S2 group exhibited a higher expression level of AIM2 than the S1 group ( $\mathrm{P}<0.05$; Fig. 3B).

Effects of sevoflurane general anesthesia during early pregnancy on the expression of $C D 45$ and $I L-1 \beta$ in the hippocampus and parietal cortex of offspring rats. In order 

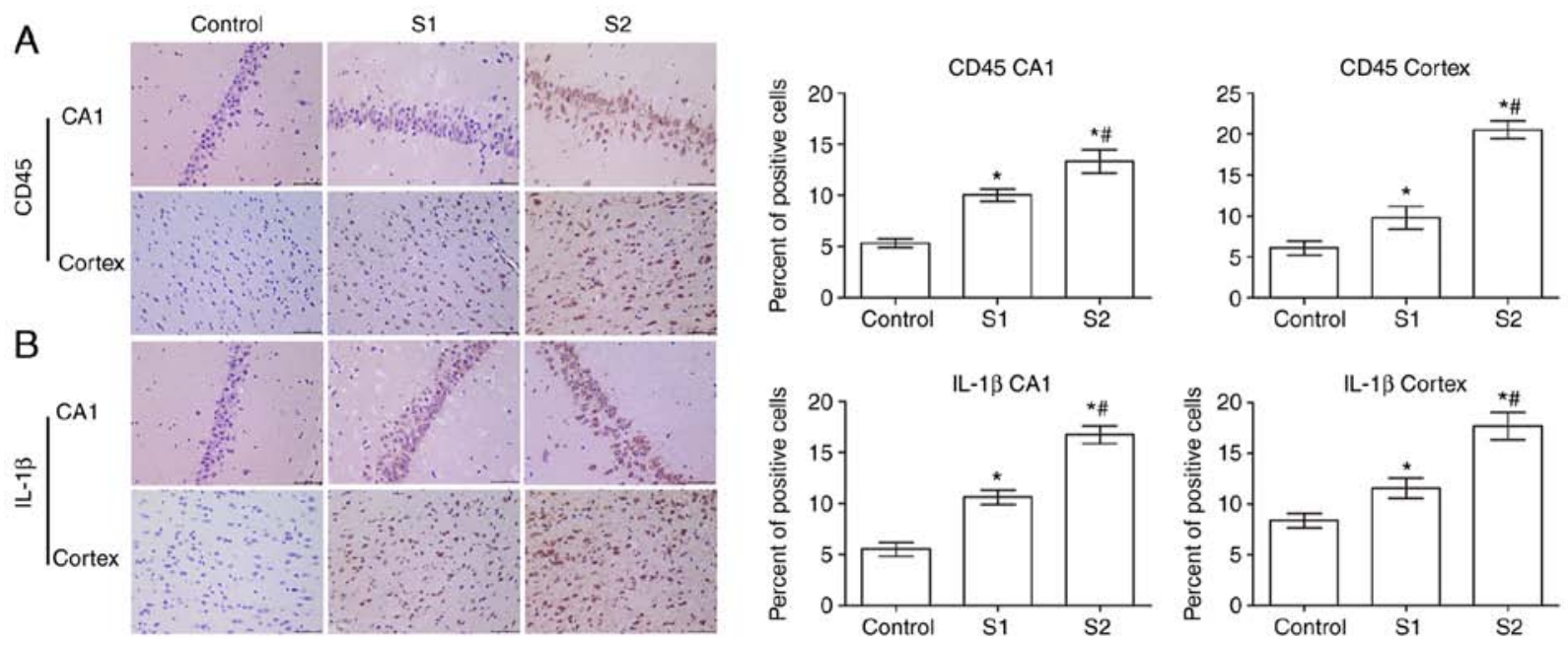

Figure 4. Immunohistochemical analysis of the expression of (A) CD45 and (B) IL-1 $\beta$ in the CA1 and cortex. Scale bar, $50 \mu \mathrm{m}$. "P<0.05 vs. the control group; ${ }^{\text {"}} \mathrm{P}<0.05$ vs. the S1 group. CA1, hippocampal cornu ammonis 1; cortex, parietal cortex; S1, 2-h sevoflurane general anesthesia; S2, 4-h sevoflurane general anesthesia.
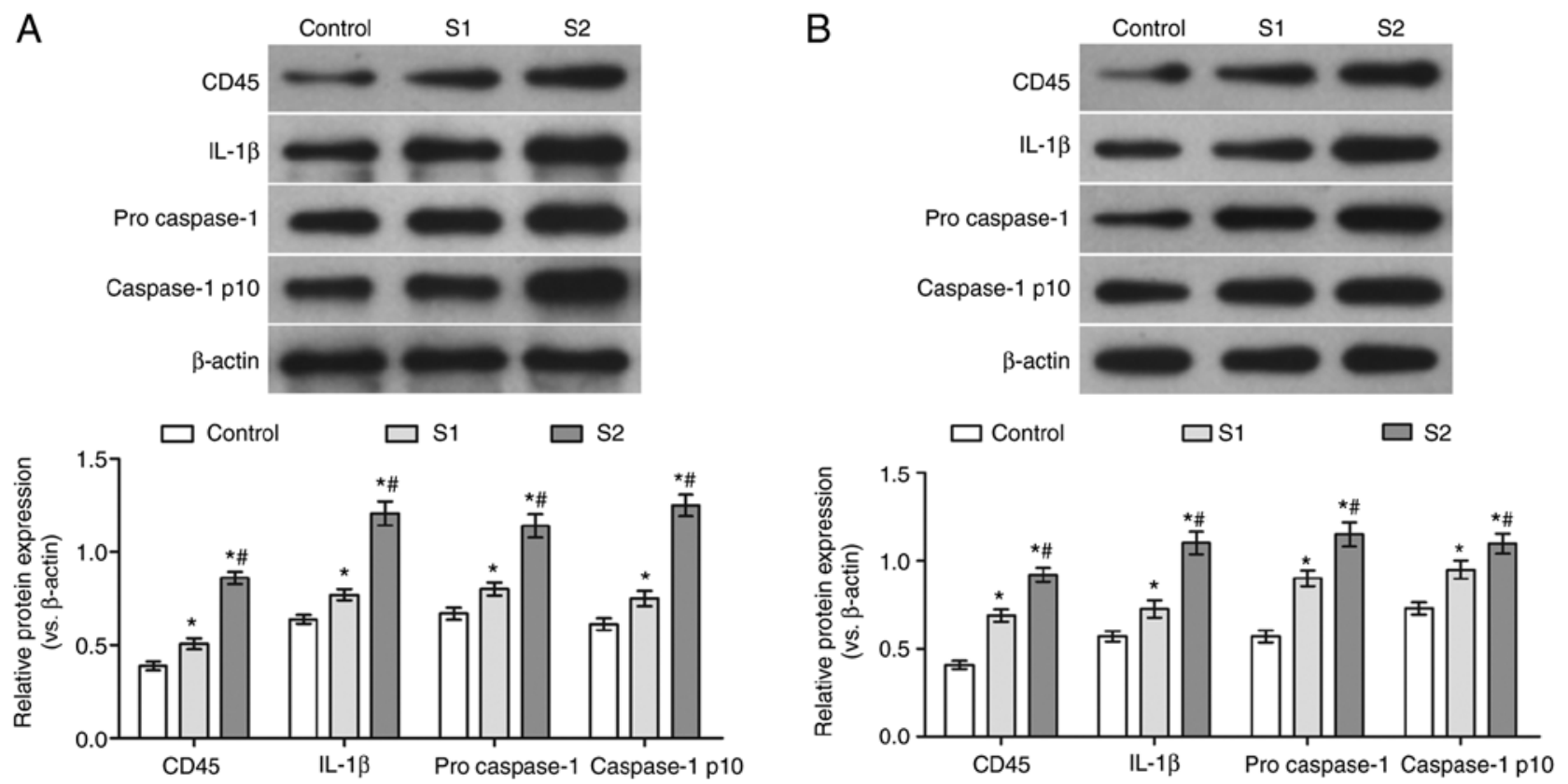

Figure 5. Western blot analysis of the protein expression levels of CD45, IL-1 1 pro-caspase-1 and caspase-1 p10 in the hippocampus (A) and parietal cortex (B). $\beta$-actin was used as the internal reference. ${ }^{*} \mathrm{P}<0.05$ vs. the control group; ${ }^{~} \mathrm{P}<0.05$ vs. the $\mathrm{S} 1$ group. $\mathrm{S} 1,2$-h sevoflurane general anesthesia; $\mathrm{S} 2$, 4-h sevoflurane general anesthesia.

to study the effect of sevoflurane general anesthesia during early pregnancy on inflammatory factors in offspring rats, the expression of CD45 and IL-1 $\beta$ in the hippocampus and parietal cortex of offspring rats was observed. As shown in Fig. 4A, the expression of CD45 in the hippocampus and parietal cortex of the $\mathrm{S} 1$ group and the $\mathrm{S} 2$ group was significantly higher than that in the control group $(\mathrm{P}<0.05)$, and the expression of $\mathrm{CD} 45$ was higher in the $\mathrm{S} 2$ group than that in the $\mathrm{S} 1$ group $(\mathrm{P}<0.05)$.

Further examination showed that compared with the control group, the expression of IL-1 $\beta$ in the hippocampus and parietal cortex of the S1 and S2 groups was significantly increased $(\mathrm{P}<0.05)$, and that this expression was significantly higher in the S2 group than that in the $\mathrm{S} 1$ group $(\mathrm{P}<0.05 ;$ Fig. $4 \mathrm{~B})$.
Effects of sevoflurane general anesthesia during early pregnancy on the protein levels of CD45, IL-1 $\beta$, pro-caspase-1 and caspase-1 p10 in the hippocampus and parietal cortex of offspring rats. To further verify the effects of sevoflurane general anesthesia during early pregnancy on the hippocampus and parietal cortex of offspring rats, the protein levels of CD45, IL- $1 \beta$, pro-caspase- 1 and caspase- 1 p10 were determined. Compared with the control group, the protein levels of CD45, IL-1 $\beta$, pro-caspase- 1 and caspase- 1 p10 in the hippocampus and parietal cortex of the $\mathrm{S} 1$ and $\mathrm{S} 2$ groups were significantly increased $(\mathrm{P}<0.05)$. Moreover, the expression of these proteins was found to be higher in the $\mathrm{S} 2$ than that in the $\mathrm{S} 1$ group $(\mathrm{P}<0.05$; Fig. 5). 


\section{Discussion}

To investigate the effects of sevoflurane exposure on the hippocampus and parietal cortex, a comparison study was designed to examine the expression of inflammation-associated proteins in rats. Previous studies on the morphological changes in neuronal cells indicated that exposure to sevoflurane during gestation was associated with offspring brain development $(13,20,21)$.

Previous research has revealed that anesthetics cause significant damage to the developing brain (22). The degree of damage is primarily dependent on the concentration of the anesthetic and the duration of exposure (23). According to previous hypotheses, in the present study, a concentration of $2 \%$ sevoflurane was administered to rats during early pregnancy (15-18). Repeated sevoflurane exposure has also been reported to suppress the proliferation of neural progenitors in offspring (24). Therefore, to investigate its effects on brain development, sevoflurane exposure times of 2 and $4 \mathrm{~h}$ were selected with reference to previous studies $(18,25)$.

AIM2 is an inflammasome cytokine that plays an essential role in the defense against bacterial and viral elements and is a reliable indicator for assessing brain changes (26). In the present study, a significant increase in AIM2 expression was observed in the offspring of the sevoflurane-treated groups. AIM2 has been reported to engage the caspse-1-activating adaptor protein to form a caspase-1-activating inflammasome (27). CD45, the lymphocyte common antigen, is a receptor-linked protein tyrosine phosphatase that plays a crucial role in leucocyte function (28). In the current study, the expression of CD45 was higher in the sevoflurane groups than that in the control group. These findings are consistent with previous studies indicating that the use of sevoflurane anesthesia in pregnant animals may affect the development of the fetal brain (29).

Abnormalities in the hippocampus and parietal cortex usually result in mental disorders such as autism spectrum disorder and neurodegeneration (30). Previous studies have shown that sevoflurane reduces the protein expression levels of caspase-3 in the fetal brain (31). IL-1 $\beta$ is a key pro-inflammatory cytokine that is essential for host defense responses to injury and infection. Caspase-1 is a functional enzyme that proteolytically cleaves other proteins, such as the precursors of the inflammatory cytokine IL-1, and thus plays a pivotal role in cellular immunity as an inflammatory response initiator. Once activated, caspase-1 usually initiates a proinflammatory response through the cleavage and thus activation of two inflammatory cytokines, IL-1 $\beta$ and IL-18 (32). In the present study, significant changes in the protein levels of CD45, IL- $1 \beta$, pro-caspase- 1 and caspase- 1 p10 were observed in the hippocampus and parietal cortex. These results suggest that sevoflurane exposure had an impact on different regions of the fetal brain.

The results of the present study indicated that damage to different regions of the brain, including the hippocampus and parietal cortex, following sevoflurane exposure may ultimately lead to functional and neurological impairments in adult offspring, which provides insights into the potential mechanisms of postoperative neurological impairment following prenatal sevoflurane exposure.
However, the present study had certain limitations. In addition to the hippocampus and parietal cortex, whether other regions of brain are affected remains to be elucidated.

In conclusion, sevoflurane general anesthesia during early pregnancy promoted the expression of AIM2 in the hippocampus and parietal cortex of offspring SD rats, and also promoted the inflammatory response in these tissues.

\section{Acknowledgements}

Not applicable.

\section{Funding}

No funding was received.

\section{Availability of data and materials}

The dataset used and/or analyzed during the current study are available from the corresponding author on reasonable request.

\section{Authors' contributions}

$\mathrm{YZ}$ and CL carried out experimental work, as well as data collection and interpretation, revised the manuscript. YZ and $\mathrm{JL}$ participated in the design and coordination of the experimental work, in addition to data acquisition. SS and WJ contributed to the study design, experimental data collection and analysis, and preparation of the manuscript. CL and JL assessed the data to ensure its legitimacy. All authors read and approved the final manuscript.

\section{Ethics approval and consent to participate}

All animal experiments were conducted following the National Institutes of Health guidelines and were reviewed and approved by the Yantaishan Hospital Animal Protection and Use Committee (approval no. 2018-10087; Yantai, China).

\section{Patient consent for publication}

Not applicable.

\section{Competing interests}

The authors declare that they have no competing interests.

\section{References}

1. Brioni JD, Varughese S, Ahmed R and Bein B: A clinical review of inhalation anesthesia with sevoflurane: From early research to emerging topics. J Anesth 31: 764-778, 2017.

2. Choi ES, Shin JY, Oh AY, Park HP, Hwang JW, Lim YJ and Jeon YT: Sevoflurane versus propofol for interventional neuroradiology: A comparison of the maintenance and recovery profiles at comparable depths of anesthesia. Korean J Anesthesiol 66: 290-294, 2014.

3. O'Farrell RA, Foley AG, Buggy DJ and Gallagher HC: Neurotoxicity of inhalation anesthetics in the neonatal rat brain: Effects on behavior and neurodegeneration in the piriform cortex. Anesthesiol Res Pract 2018: 6376090, 2018. 
4. Ji MH, Wang XM, Sun XR, Zhang H, Ju LS, Qiu LL, Yng JJ, Jia M, Wu J and Yang J: Environmental ehrichment ameliorates neonatal sevoflurane exposure-induced cognitive and synaptic plasticity impairments. J Mol Neurosci 57: 358-365, 2015.

5. Wu J, Yu J, Xie P, Maimaitili Y, Wang J, Yang L, Ma H, Zhang X, Yang Y and Zheng H: Sevoflurane postconditioning protects the myocardium against ischemia/reperfusion injury via activation of the JAK2-STAT3 pathway. PeerJ 5: e3196, 2017.

6. Juhasz-Boss I, Solomayer E, Strik M and Raspe C: Abdominal surgery in pregnancy-an interdisciplinary challenge. Dtsch Arztebl Int 111: 465-472, 2014.

7. Olutoye OA, Baker BW, Belfort MA and Olutoye OO: Food and Drug Administration warning on anesthesia and brain development: Implications for obstetric and fetal surgery. Am J Obstet Gynecol 218: 98-102, 2018.

8. Malhotra A, Yosh E and Xiong M: Propofol's effects on the fetal brain for non-obstetric surgery. Brain Sci 7: 107, 2017.

9. Walkden GJ, Pickering AE and Gill $\mathrm{H}$ : Assessing long-term neurodevelopmental outcome following general anesthesia in early childhood: Challenges and opportunities. Anesth Analg 128: 681-694, 2019.

10. Vutskits L and Xie Z: Lasting impact of general anaesthesia on the brain: Mechanisms and relevance. Nat Rev Neurosci 17: 705-717, 2016

11. Jevtovic-Todorovic V: Exposure of developing brain to general anesthesia: What is the animal evidence? Anesthesiology 128 832-839, 2018.

12. Bi C, Cai Q, Shan Y, Yang F, Sun S, Wu X and Liu H: Sevoflurane induces neurotoxicity in the developing rat hippocampus by upregulating connexin 43 via the JNK/c-Jun/AP-1 pathway. Biomed Pharmacother 108: 1469-1476, 2018.

13. Song R, Ling X, Peng M, Xue Z, Cang J and Fang F: Maternal sevoflurane exposure causes abnormal development of fetal prefrontal cortex and induces cognitive dysfunction in offspring. Stem Cells Int 2017: 6158468, 2017.

14. National Institutes of Health (NIH) guidelines revised in 1996. https://nihrecord.nih.gov/.

15. Huang H, Liu CM, Sun J, Jin WJ, Wu YQ and Chen J: Repeated $2 \%$ sevoflurane administration in 7-and 60-day-old rats: Neurotoxicity and neurocognitive dysfunction. Aneasthesist 66 : 850-857, 2017

16. Guo Z, Zhao F, Wang Y, Wang Y, Geng M, Zhang Y, Ma Q and $\mathrm{Xu} \mathrm{X}$ : Sevoflurane Exerts an Anti-depressive Action by Blocking the HMGB1/TLR4 Pathway in Unpredictable Chronic Mild Stress Rats. J Mol Neurosci 69: 546-556, 2019.

17. $\mathrm{Yu} X$, Zhang $\mathrm{F}$ and Shi J: Neonatal exposure to sevoflurane caused cognitive deficits by dysregulating SK 2 channels and GluA2-lacking AMPA receptors in juvenile rat hippocampus. Neuropharmacology 141: 66-75, 2018.

18. Zheng SQ, An LX, Cheng X and Wang YJ: Sevoflurane causes neuronal apoptosis and adaptability changes of neonatal rats. Acta Anaesthesiol Scand 57: 1167-1174, 2013.

19. Zhang Y, Yang F, Gao Y, Shan Y, Dong Y and Liu H: Neuroglobin protects offspring rats from neuronal damage induced by sevoflurane exposure to pregnant rats by inhibiting endogenous apoptosis. Int J Dev Neurosci 76: 17-24, 2019.

20. Wu Z, Li X, Zhang Y, Tong D, Wang L and Zhao P: Effects of sevoflurane exposure during mid-pregnancy on learning and memory in offspring rats: Beneficial effects of maternal exercise. Front Cell Neurosci 12: 122, 2018.
21. Fang F, Song R, Ling X, Peng M, Xue Z and Cang J: Multiple sevoflurane anesthesia in pregnant mice inhibits neurogenesis of fetal hippocampus via repressing transcription factor Pax6. Life Sci 175: 16-22, 2017.

22. Sharma HS, Muresanu DF, Nozari A, Castellani RJ, Dey PK, Wiklund L and Sharma A: Anesthetics influence concussive head injury induced blood-brain barrier breakdown, brain edema formation, cerebral blood flow, serotonin levels, brain pathology and functional outcome. Int Rev Neurobiol 146: 45-81, 2019.

23. Rocha TL, Dias-Junior CA, Possomato-Vieira JS, Goncalves-Rizzi VH, Nogueira FR, de Souza KM, Braz LG and Braz MG: Sevoflurane induces DNA damage whereas isoflurane leads to higher antioxidative status in anesthetized rats. Biomed Res Int 2015: 264971, 2015

24. Zhang MQ, Ji MH, Zhao QS, Jia M, Qiu LL, Yang JJ, Peng YG, Yang JJ and Martynyuk AE: Neurobehavioural abnormalities induced by repeated exposure of neonatal rats to sevoflurane can be aggravated by social isolation and enrichment deprivation initiated after exposure to the anaesthetic. Br J Anaesth 115: $752-760,2015$

25. Guo SB, Liu LD, Wang C, Jiang Q, Dong YX and Tian Y: Repeated exposure to sevoflurane impairs the learning and memory of older male rats. Life Sci 192: 75-83, 2018.

26. Ge X, Li W, Huang S, Yin Z, Xu X, Chen F, Kong X, Wang H, Zhang $J$ and Lei P: The pathological role of NLRs and AIM2 inflammasome-mediated pyroptosis in damaged blood-brain barrier after traumatic brain injury. Brain Res 1697: 10-20, 2018.

27. Lam HYP, Chen TT, Chen CC, Yang TH, Cheng PC and Peng SY: Angiostrongylus cantonensis activates inflammasomes in meningoencephalitic BALB/c mice. Parasitol Int 77: 102119, 2020.

28. Rheinländer A, Schraven B and Bommhardt U: CD45 in human physiology and clinical medicine. Immunol Lett 196: 22-32, 2018.

29. Hirotsu A, Iwata Y, Tatsumi K, Miyai Y, Matsuyama T and Tanaka T: Maternal exposure to volatile anesthetics induces IL-6 in fetal brains and affects neuronal development. Eur J Pharmacol 863: 172682, 2019.

30. Gilsoul M, Grisar T, Delgado-Escueta AV, de Nijs L and Lakaye B: Subtle brain developmental abnormalities in the pathogenesis of Juvenile Myoclonic epilepsy. Front Cell Neurosci 13: 433, 2019.

31. Perez-Zoghbi JF, Zhu W, Grafe MR and Brambrink AM: Dexmedetomidine-mediated neuroprotection against sevoflurane-induced neurotoxicity extends to several brain regions in neonatal rats. Br J Anaesth 119: 506-516, 2017.

32. Malik A and Kanneganti TD: Inflammasome activation and assembly at a glance. J Cell Sci 30: 3955-3963, 2017.

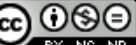

This work is licensed under a Creative Commons Attribution-NonCommercial-NoDerivatives 4.0 International (CC BY-NC-ND 4.0) License. 